\title{
Peer To Peer Content Sharing On Wi-Fi Network For Smart Phones
}

\author{
Pradnya Mate ${ }^{1}$, Snehal Dhamale ${ }^{2}$, Pranita Ohal ${ }^{3}$, Ruchi Pawar ${ }^{4}$ \\ Department of Computer Engineering, Sinhgad College of Engineering, Vadgaon (Bk), Pune \\ University of Pune, India.
}

\begin{abstract}
Peer-to-Peer (P2P) communications and its applications have become conventional architecture in the wired network environment. However, they have not been effectively adapted to the ensemble mobile environment which composed of various devices such as smart mobiles devices, laptops, and device with embedded software. In P2P systems, each node can act as a client and as a server at the same time and shares with others its own data. Our contribution consists in designing, implementing and testing a BitTorrent like application adapted to wireless ad-hoc networks of Android Mobile Phones. The employment of P2P protocols and applications in the platform of the ensemble mobile environment is becoming a promising solution which permits a wide number of users to share their own contents (data, audio, video, etc.) and communicate with each other without using costly and centralized network infrastructure. By using a central server, the peers will be able to get the information about the contents of other peers. Even in the absence of the Internet, the mobile devices provide users with the potential opportunity to connect in ad-hoc manner via short range wireless protocol such as Wi-Fi.
\end{abstract}

Keywords- Peer to peer computing, mobile ad hoc networks, wireless communication, Wi-Fi, multimedia communication.

\section{INTRODUCTION}

In the recent years, Peer-to-Peer (P2P) communications and its applications have become conventional architecture in the wired network environment. However, they have not been effectively adapted to the ensemble mobile environment which composed of various devices such as smart mobile devices, laptops, and devices with embedded software. In P2P systems, each node can act as a client and as a server at the same time and shares with others its own data ${ }^{[1][6]}$.

Our contribution consists in designing, implementing and testing a Bit Torrent like application adapted to wireless ad-hoc networks of Android Mobile Phones ${ }^{[7]}$.

They have become highly-capable multimedia devices, with embedded video cameras, and many users enjoy sharing the multimedia content that they capture. For example, as part of a home entertainment system, the user of a smart phone may wish to upload user-generated content such as newly-taken videos to another smart phone or tablet device, to a media player connected to a TV, or perhaps to a wireless picture frame. Several users may be interested in automatically receiving multimedia from one particular source ${ }^{[3][4][5]}$. The problem is that when employing a $3 \mathrm{G}$ wireless data connection and uploading to a traditional web server, these transfers can quickly become prohibitively expensive. Users are typically subject to wireless data plans with finite usage limits and steep overage charges. The alternative of uploading content through a wired USB connection is cumbersome and delays the user experience ${ }^{[1][2]}$.

A promising solution is to employ peer-to-peer sharing among smart phones, in order to consume free peer-to-peer wireless links versus expensive packet data networks for file sharing purposes. For instance, a camera-enabled device could publish new content and advertise it through a service that the other devices in the home would subscribe to. Through peer -to- peer connectivity, the participating hardware devices could then share the multimedia resources back and forth without the use of any intermediary hosts, and not be subject to billing $^{[8][9]}$.

\section{Problem Statement}

To develop a system for providing peer-to-peer content sharing on Wi-Fi network for Smart Phones. Peer-to-peer model faces unique challenges in the mobile context - limitations on processing power, on-board device memory, wireless data bandwidth, available battery energy. To employ peer-to-peer sharing among smart phones to consume free peer-to-peer wireless links. 


\subsection{Bluetooth Technology:}

\section{TECHNOLOGY DETAILS}

Bluetooth is a technology that allows device to communicate and share data over short distances without wires. It is a proprietary open wireless technology standard for exchanging data from fixed and mobile devices, creating personal area network (PANs) with high levels of security. It is a standard wire-replacement communication protocol primarily designed for low power consumption, with a short range based on low-cost transceiver microchips in each device. Anyone who joins the Bluetooth Special Interest Group and adheres to well outlined standards can create Bluetooth devices. While Bluetooth technology is prevalent

in devices that need to transfer small amount of data, the technology's limitations have prevented it from becoming the de-facto wireless technology. Devices such as mobile phones, laptops, PCs, printers, digital cameras, and video game console can connect to each other, and exchange information. Bluetooth is only used for relatively short distances, like a few meters.

Followings are the limitations of Bluetooth:

A. The rate of data transfer between Bluetooth device is about 3 megabits per second. This is significantly lower than Wi-Fi.

\section{B. The range of communication is limited.}

\subsection{Wi-Fi Technology:}

Wi-Fi technology is an alternative to wired technology, which is commonly used for connecting devices in wireless mode. Wi-Fi (Wireless Fidelity) is a generic term that refers to IEEE 802.11 communication standards for Wireless Local Area Networks. It uses radio technologies to transmit and receive data at high speed.

It is popular technology that allows an electronic device to exchange data wirelessly (using radio waves) over a computer network, including high speed Internet connections. Wi-Fi provides its users with the liberty of connecting to the internet from any place within Wi-Fi area without the hassles of plugging in wires. Wi-Fi location where the users can connect to the wireless network is called a Wi-Fi hotspot. Through the Wi-Fi hotspot, the users can even enhance their home business, as accessing information through Wi-Fi is simple. Accessing a wireless network through hotspot in some cases is cost-free.

Following are the Advantages of Wi-Fi over Bluetooth:

1. The most prominent advantage which Wi-Fi has over Bluetooth is that Wi-Fi operates at a much faster rate of $11 \mathrm{mbps}$, whereas Bluetooth only operates at a much slower rate of around $720 \mathrm{kbps}$. This makes Bluetooth too slow for video transfers or moving large amounts of large photo images from a digital camera.

2. Wi-Fi is also designed to link up entire networks, rather than computer to computer.

\subsection{Peer to Peer Technology:}

A peer to peer (abbreviated to P2P) computer network is one in which each computer in network can act as a client or server for the other computers in the network, allowing shared access to various resources such as files, peripherals and sensors without the need for a central server. P2P network can be set up within the home, a business, or over the Internet. Each network type requires all computers in the network to use same or a compatible program to connect to each other and access files and other resources found on the other computer.

P2P networks can be used for sharing contents such as audio, video, data, or anything in digital format.

$\mathrm{P} 2 \mathrm{P}$ is a distributed application architecture that partitions tasks or workloads among peers. Peers are equally previledged participants in the application. Each computer in a network is refered to as node. The owner of each computer on a P2P network would set aside a portion of its resources - such as processing power, disk storage, or network bandwidth - to be made directly available to other network participant, without the need for central coordination by servers or stable hosts. With this model, peers are both suppliers and consumers of resources, in contrast to the traditional client-server model where only the server supply (send), and client consume (receive).

\section{SYSTEM ARCHITECTURE}

System comprises of following seven modules as shown in Figure 1:

\subsection{Client Side}

A. Network Discovery Manager: It is responsible for handling the connectivity of the system, both detecting Wi-Fi Access Points and determining when peers have become disconnected.

B. Communication Manager: It deals with sending request to the server for searching contents and receiving list of peers containing required contents.

C. File System Reader/Writer: It is responsible for reading files and writing files to the system.

D. Private Folder Locker: It allows user to make content available to other peers. 


\subsection{Server Side}

A. Transaction Tracker: It keeps the track of transactions carried out by users.

B. Request Response Handler: It handles request from user and provides response accordingly.

C. GUI : It provides interaction to administrator.

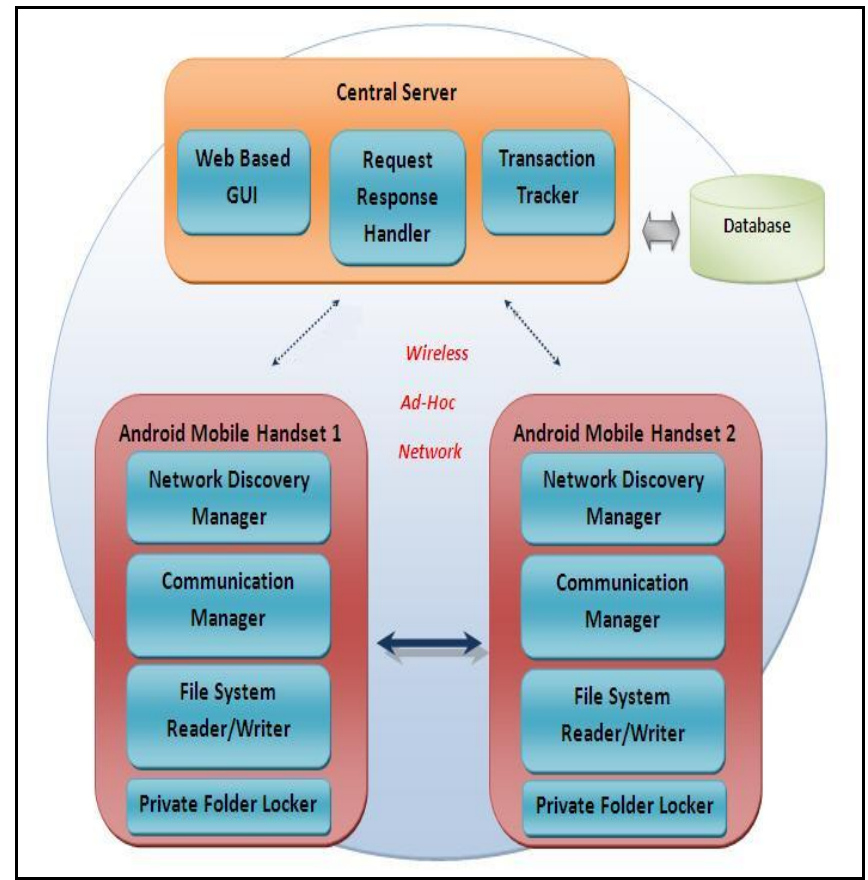

Fig. 1 System Architecture

\section{IMPLEMENTATION}

The employment of P2P protocols and applications in the platform of the ensemble mobile environment is becoming a promising solution which permits a wide number of users to share their own contents (data, audio, video, etc) and communicate with each other without using costly and centralized network infrastructure. By using a central server, the peers will be able to get the information about the contents of other peers. When receiver peer requests for the required content, the request will pass to the central server. Then central server searches for the peers where the requested content is available and gives the metadata of that peers to the receiver peer. After that receiver peer makes the connection with the sender peer and then peer to peer content sharing takes place.

The mobile devices provide users with the potential opportunity to connect in ad-hoc manner via short range wireless protocol such as $\mathrm{Wi}-\mathrm{Fi}$.

The system implementation consists of five modules:
a. Sharable Folder
b. Request Response Handler
c. Searching
d. Downloading
e. Chat Application

Sharable Folder:

User will be able to put files in the Sharable Folder that he or she wants to share on the network with other users in the same network.

Request Response Handler:

Request Response Handler will deal with the handshaking activity which is involved in the connection establishment initially.

Searching:

Searching module will be responsible for finding out whether any of the peers in the network contains the required file. File will be searched in the network based on its name. 
Downloading:

Server will send the search results to the requesting peer which contains file name and IP address of the peer having the requested file. Further the Downloading module will be involved in the actual process of downloading the requested file.

Chat Application:

Our system will be provided with the Chatting Application which is an extension of Peer to Peer communication. As there is no server involvement in the communication among two peers, Chatting Application will be pure Peer to Peer Communication.

\section{CONCLUSION AND FUTURE WORK}

We have proposed a peer-to-peer model that permits efficient file sharing between mobile smart phones over a low-cost transport. The employment of $\mathrm{P} 2 \mathrm{P}$ protocols and applications in the platform of the ensemble mobile environment is becoming a promising solution which permits a wide number of users to share their own contents (data, audio, video, etc.) with each other without using costly and centralized network infrastructure Small segments are desirable when multiple clients are involved, as they allow for increased parallelism of downloads.

The use of socket for content sharing is more optimal, as it only requires two sockets: one for downloading, and one for uploading, for each peer, regardless of the number of peers within the network area. Peer-to-peer sharing allows for efficient content distribution using low-cost links that do not impose a load on the mobile carrier infrastructure.

It is expected that the proposed protocol will allow smart device users to communicate among each other in real time by using various P2P applications. From a utilization point of view, we plan to combine this work with other proximity-based services such as medical or social ones.

This paper can be considered for future enhancements in order to provide and maintain quality of service for an application during transmission of data for the purpose of reliability and efficiency.

\section{REFERENCES}

[1] "Framework for Enhancing P2P Communication Protocol on Mobile Platform", Waheb A. Jabbar, M. Ismail, R. Nordin.

[2] “Architectural Model for Wireless Peer-to-Peer (WP2P) File Sharing for Ubiquitous Mobile Devices", O.O.Abiona, A.I.Oluwaranti T.Anjali,C.E.Onime,E.O.Popoola,G.A.Aderounmu,A.O.Oluwatope and L.O.Kehinde, 978-1-4244-3355-1/09/\$25.00 @2009 IEEE.

[3] "BlueStreaming:Towards Power-Efficient Internet P2P Streaming to Mobile Devices",You Liu,Fei Li, Lei Guo, Yang Guo, Songqing Chen. MM'11, November 28-December 1, 2011, Scottsdale, Arizona, USA.Copyright 2011 ACM 978-1-4503-0616-4/11/11 ...\$10.00.

[4] "Broadcast-Based Peer-To-Peer Collaborative Video

[5] Streaming Among Mobiles",Man-Fung Leung and S.-H Gray Chan,2007. IEEE TRANSACTIONS ON BROADCASTING, VOL. 53, NO. 1, MARCH 2007

[6] "Quality of Data Delivery in Peer-to-Peer Video Streaming",XIAOSONG LOU and Kai HWANG,University of Southrn California,ACM Transactions on Multimedia Computing,Communication and Applications, Vol.8S,No.1,Article 12,Publication date:February 2012.

[7] "Peer to Peer Content Sharing on Ad Hoc Networks of Smartphones", Piotr K.Tysowski, Pengxiang Zhao, Kshirasagar Naik. 978-14577-9538-2/11/\$26.00 @2011 IEEE.

[8] "Clustering and Sharing Incentives in BitTorrent Legout,I.N.R.I.A.,SophiaAntipolis,France,arnaud.legout@sophia.inria.fr,Nikitas Liogkas ,Eddie Kohler,Lixia Zhang,University of California,Los Angeles,CA USA,\{nikitas,kohler,lixia\}@cs.ucla.edu. SIGMETRICS’07, June 12-16, 2007, San Diego, California, USA ,Copyright 2007 ACM 978-1-59593-639-4/07/0006 ...\$5.00.

[9] "P2P File Sharing on Mobile Phones: Design and Implementation of a Prototype", Claudio E. Palazzi, Armir Bujari, Emanuele Cervi ,Dipartimento di Matematica Pura e Aniversità di Padova, Padova,Italycpalazzi@math.unipd.it,abujari@studenti.math.unipd.it, ecervi@ studenti.math.unipd.it

[10] “A Peer-to-Peer File Resource Sharing System for Mobile Devices “,Chao-Tung Yang, Chun-Jen Chen, Hung-Yen Chen ,HighPerformance Computing Laboratory ,Department of Computer Science and Information Engineering, Tunghai University, Taichung, 40704, Taiwan R.O.C. ctyang@thu.edu.tw, 978-0-7695-3177-9/08 \$25.00 @ 2008 IEEE DOI 10.1109/GPC.WORKSHOPS.2008.75

[11] "Creating a mobile P2P file sharing environment over Bluetooth",Gerard McNamara,Yanyan Yang, 978-1-4244-2020-9/08/\$25.00 02008 IEEE . 